\title{
Acidentes de trabalho atendidos em serviço de emergência
}

\author{
Occupational injuries treated \\ in an emergency room
}

Paulo Sérgio de Andrade Conceição 1

Itatyane Bispo de Oliveira Nascimento 1

Patrícia Silva Oliveira 1

Maria Ruth Moreira Cerqueira 1

1 Centro de Estudos da Saúde do Trabalhador, Secretaria da Saúde do Estado da Bahia. Rua Pedro Lessa 123,

Salvador, $B A$ 40110-050, Brasil. paulo-conceicao@uol.com.br itatyane@bol.com.br
Abstract In Brazil, work-related injuries are only reported to the National Social Security data system. Therefore, records are limited to formally hired workers, who represent less than half of the active work force. In this cross-sectional study, cases of work-related injuries were identified and interviewed in an emergency room in the city of Salvador, capital of Bahia State, Brazil, to estimate their frequency and characteristics. Work-related injuries accounted for $31.6 \%$ of all injuries from external causes $(n=215)$. Only $36.8 \%$ of these patients reported having a formal job contract, and of these, fewer than half (45.5\%) had their injuries reported to the Social Security data system, which indicates extensive underreporting of such important work-related health events, even in the formal sector of the economy. Medical records from emergency rooms can be an important source of information on work-related accidents, and health surveillance needs to be enforced in such health care services. This can also be an important step in obtaining a more complete picture of the occurrence of work-related accidents in Brazil.

Key words Occupational Accidents; Emergencies; Cross-Sectional Studies; Underregistration

Resumo No Brasil, acidentes de trabalho (AT) são notificados apenas para a Previdência Social, restringindo-se, portanto, aos trabalhadores com carteira assinada, que representam, atualmente, menos da metade da força de trabalho ocupada. Neste estudo transversal, pretende-se descrever os acidentes de trabalho e estimar a sua freqüência dentre os casos atendidos num serviço de emergência de Salvador, Bahia, Brasil. Identificou-se 215 pessoas atendidas por causas externas, das quais 68 (31,6\%) eram AT. Verificou-se que 36,8\% dos acidentados tinham carteira assinada, e dentre esses, apenas 45,5\% tiveram notificação para a Previdência, o que indica o alto nível de sub-registro dos AT, mesmo entre os trabalhadores do setor formal. Registros de serviços de emergência podem ser importante fonte de informação dos acidentes de trabalho, e a extensão das ações de vigilância para estes locais poderá ser um passo importante para um melhor conhecimento da magnitude de ocorrência desses eventos na população.

Palavras-chave Acidentes de Trabalho; Emergências; Estudos Transversais; Sub-Registro 


\section{Introdução}

No Brasil, a realidade dos acidentes de trabalho (AT) é conhecida apenas parcialmente, pois a sua notificação é limitada à Previdência Social. Essa notificação é feita através da Comunicação de Acidente de Trabalho (CAT) para os empregados com carteira assinada, excluindo-se os trabalhadores domésticos. Segundo a Fundação Instituto Brasileiro de Geografia e Estatística (IBGE, 2000), em 1999, o número de trabalhadores com carteira assinada era de 19,6 milhões, enquanto a população ocupada (incluindo os empregados sem carteira, estatutários, trabalhadores domésticos e aqueles do chamado setor informal) totalizava 71,6 milhões. Portanto, a notificação dos acidentes de trabalho limita-se a pouco mais de um quarto do total de trabalhadores.

Os acidentes de trabalho são ocorrências relevantes dentre as causas externas atendidas em serviços de saúde. Num estudo realizado no Rio de Janeiro por Deslandes (1999), em dois hospitais municipais de emergência, a proporção de acidentes de trabalho dentre as causas externas foi de 18,7\% num hospital e 15,0\% no outro. Sabe-se por outro lado, que mesmo os acidentes do trabalho em empregados com carteira assinada deixam de ser notificados. Num inquérito domiciliar realizado em São Paulo (Barata et al., 2000), observou-se um sub-registro médio dessas ocorrências de $75,0 \%$.

Este estudo teve o objetivo de avaliar a proporção dos acidentes de trabalho dentre os atendimentos por causas externas num serviço de emergência e descrever o perfil dessas ocorrências em relação a características dos acidentados, causas das ocorrências, ramos de atividade das empresas e os principais diagnósticos, na perspectiva de implementação da sua vigilância, considerando-se que grande parte dos trabalhadores que sofre acidentes de trabalho deve ser encaminhada aos serviços de emergência, independentemente do seu tipo de vínculo empregatício.

\section{Métodos}

Este é um estudo transversal conduzido com a demanda atendida na principal unidade de emergência, localizada em Salvador, capital do Estado da Bahia, Brasil.

A definição utilizada para as causas externas foi a que está expressa na Política Nacional de Redução da Morbimortalidade por Acidentes e Violências, aprovada através da Portaria do Ministério da Saúde (MS) de número 737 de
16 de maio de 2001 (Brasil, 1997), que diz: "Os acidentes e as violências configuram, assim, um conjunto de agravos à saúde, que pode ou não levar a óbito, no qual se incluem as causas ditas acidentais - devidas ao trânsito, trabalho, quedas, envenenamentos, afogamentos e outros tipos de acidentes - e as causas intencionais (agressões e lesões autoprovocadas). Esse conjunto de eventos consta na Classificação Internacional de Doenças (CID) sob a denominação de causas externas. Quanto à natureza da lesão, tais eventos e ou agravos englobam todos os tipos de lesões e envenenamentos, como ferimentos, fraturas, queimaduras, intoxicações, afogamentos entre outros".

Os registros hospitalares não estavam bem organizados, o que dificultou a identificação do tamanho adequado da amostra. Optou-se por fazer um levantamento dos atendimentos realizados durante oito plantões diurnos das 07:00 às 19:00, em dias consecutivos, excluindo-se o final de semana. Assumiu-se, portanto, que esses representariam o total de atendimentos nos dias úteis e durante o dia. Esses horários foram intencionalmente escolhidos, considerando que a maior parte das pessoas trabalha durante este tempo, quando ocorre a maioria dos acidentes de trabalho.

Foram coletadas informações dos indivíduos a partir de dez anos de idade, que corresponde ao grupo populacional em idade ativa, de acordo com a classificação do IBGE.

Um questionário único foi elaborado considerando as principais causas externas, contendo os dados pessoais e para caracterização do evento. Este foi testado no próprio Hospital onde foi realizada a coleta. Havia duas perguntas que permitiam ao entrevistador definir o evento como AT ou não: "Estava numa atividade de trabalho ou a serviço quando ocorreu o acidente?" e "O acidente ocorreu quando você estava indo ou voltando do trabalho?" No caso afirmativo, preenchia uma ficha complementar onde constava o tipo de vínculo de trabalho, o local de trabalho (município e ramo de atividade) e diagnóstico das lesões.

Foi realizado um treinamento para padronização da coleta, e o levantamento dos dados foi realizado por estudantes de Medicina, estagiários de Serviço Social e Terapia Ocupacional, e funcionários do Centro de Estudos da Saúde do Trabalhador (CESAT) da Secretaria Estadual de Saúde, supervisionados diariamente pela coordenação da pesquisa.

A rotina do Hospital compreendia a triagem dos pacientes na recepção, e o atendimento inicial de todos os casos de causas externas ocorre numa única sala, de onde os pacientes são li- 
berados de alta ou encaminhados para o "ortotrauma”, centro cirúrgico ou enfermaria, a depender da necessidade. Os entrevistadores permaneciam na sala de atendimento inicial e com o consentimento dos pacientes, entrevistavam todos os que lá tivessem sido atendidos no período da coleta. Havia sempre uma dupla de coleta em cada turno, visando evitar o não registro de alguma ocorrência de causa externa atendida, e para possibilitar checar noutros setores a eventualidade de atendimento de algum paciente de causa externa porventura não encaminhado ao setor específico.

O diagnóstico das lesões foi coletado da ficha de atendimento, conforme a anotação médica. A classificação dos ferimentos em leves e graves seguiu a proposta do Programa de Saúde do Trabalhador da Zona Norte de São Paulo, que desenvolveu o sistema de informações das comunicações de acidente de trabalho (SISCAT), sendo basicamente definido em termos dos efeitos sobre a saúde dos trabalhadores. Exemplificando, um ferimento sem menção à lesão de tendões ou vísceras é considerado $l e$ $v e$, já com menção à lesão de tendões ou vísceras é considerado grave.

Dentre o total de atendimentos por causas externas coletados, foram selecionados para este estudo os classificados como acidentes de trabalho. Todos os empregados domésticos foram considerados informais, pois pela legislação previdenciária, não se registra acidentes de trabalho para essa categoria.

A análise foi realizada através do programa Epi Info versão 6. Descreveram-se as freqüências simples das variáveis sexo, idade, escolaridade, ocupação, tipo de vínculo de trabalho, ramo de atividade e diagnóstico.

\section{Resultados e discussão}

Identificaram-se 215 pessoas cujos eventos que os levaram ao serviço foram diagnosticados como Causas Externas (capítulo XIX da CID 10 OMS, 1995). Desses, 68 (31,6\%) foram classificados como AT. Os acidentes de trabalho típicos representaram $77,9 \%$ contra $22,1 \%$ de acidentes de trajeto.

Cerca de $90 \%$ dos acidentados do trabalho eram do sexo masculino, enquanto a média de idade foi de 31,1 anos, variando de 18 a 54 anos. Cerca de $69,1 \%$ não havia completado o primeiro grau, desses $5,9 \%$, eram analfabetos. As principais ocupações dos acidentados foram pedreiro (10,3\%) e carpinteiro $(8,8 \%)$.

Dos acidentados no trabalho, 41,2 \% eram autônomos (contribuintes ou não da Previdên- cia), $36,8 \%$ tinham carteira assinada, $14,7 \%$ eram empregados, porém sem carteira de trabalho assinada e 7,3\% eram empregados domésticos (com ou sem carteira de trabalho assinada). Não houve acidentes em trabalhadores estatutários. A proporção de trabalhadores empregados com carteira assinada, dentre os acidentados do trabalho encontrada neste estudo, foi semelhante à participação dos empregados com carteira assinada dentre o total de trabalhadores da população ocupada.

Os principais ramos de atividade das empresas onde os acidentados trabalhavam podem ser vistos no Figura 1.

Foram coletadas informações sobre o município onde o trabalhador residia, o local do acidente, e o de trabalho, sendo o Município de Salvador o principal local, representando, respectivamente, $77,9 \%, 70,6 \%$ e $70,6 \%$. Os demais municípios foram, em geral, da Região Metropolitana de Salvador.

Para a descrição das principais causas dos acidentes, separaram-se os típicos dos de trajeto (Tabela 1).

As principais partes do corpo atingidas foram a mão, 27,3\%, a cabeça, 19,5\%, os membros inferiores (exclusive o pé), 15,6\% e múltiplas localizações com $11,7 \%$. Os principais diagnósticos registrados foram: ferimentos leves, $29,8 \%$, fraturas e luxações, $16,4 \%$, ferimentos graves, $13,4 \%$ e politraumatismo, $10,4 \%$. Não houve variações importantes desses diagnósticos quan-

Figura 1

Principais ramos de atividades das empresas onde os acidentados trabalhavam.



* Incluso bares, restaurantes e similares. 
Tabela 1

Principais causas dos acidentes típicos e de trajeto.

\begin{tabular}{lcccc}
\hline Causas & \multicolumn{2}{c}{$\begin{array}{c}\text { Acidente típico } \\
\text { Número }\end{array}$} & $\begin{array}{c}\text { Acidente de trajeto } \\
\text { Púmero }\end{array}$ & Percentual \\
\hline $\begin{array}{l}\text { Queda de peso } \\
\text { sobre o corpo }\end{array}$ & 10 & 18,9 & - & - \\
Quedas1 & 08 & 15,1 & 03 & 20,0 \\
Máquinas & 08 & 15,1 & - & - \\
Acidentes de trânsito & 07 & 13,2 & 07 & 46,7 \\
Outros & 20 & 37,7 & 05 & 33,3 \\
Total & 53 & 100,0 & 15 & 100,0 \\
\hline
\end{tabular}

1 Quedas de altura ou sem diferença de nível.

do comparados os municípios de ocorrência, incluindo Salvador.

Comparando-se os trabalhadores de acordo com seu tipo de vínculo ao trabalho, analisando-se os acidentados separadamente em formais e informais, verificou-se uma menor participação feminina e menor proporção de acidentes de trajeto dentre os trabalhadores formais. Com relação ao nível de escolaridade, embora pouco inferior entre os trabalhadores informais, ressalta-se a baixa escolaridade nos dois grupos. Quanto aos ramos de atividade onde trabalhavam os acidentados, observou-se que entre os formais aumenta a participação da construção civil, do comércio e da indústria da transformação (Tabela 2).

Um dos primeiros estudos produzidos no Brasil, que contempla dados de AT no setor informal da economia, foi um inquérito domiciliar realizado em São Paulo (Barata et al., 2000), com o relato de uma maior incidência de acidentes de trabalho entre autônomos do que entre os assalariados, no período recordatório de um ano.

Dentro do Sistema Único de Saúde (SUS) têm sido discutidas alternativas para se conhecer melhor essa realidade, como a estratégia de notificação obrigatória dos acidentes graves e fatais, estimular o registro dos acidentes fatais a partir das Declarações de Óbito (DO) no Sistema de Informações sobre Mortalidade (SIM), ou a morbidade por acidentes de trabalho nas internações, através do Sistema de Informações Hospitalares (SIH).

Embora de grande importância, essas iniciativas ainda não têm alcançado os seus objetivos porque não se difundiram pelo país, ou pelo inadequado preenchimento dos formulários dos sistemas de informação, principalmente no que se refere ao registro da ocupação e das circunstâncias de ocorrência das causas exter- nas (Beraldo et al., 1993; Carvalho, 1997; Drumond Jr., 1999; Waldman \& Mello-Jorge, 1999).

O SIH reúne os dados de todas as internações pagas pelo SUS, nos hospitais próprios ou conveniados, e representou cerca de 12 milhões de internações por ano, em 1998 e 1999. Estimase que esse total represente cerca de $80 \%$ de todas as internações realizadas no país (Mendes, 2000). A partir do ano de 1998, em decorrência da Portaria 142/1997 do MS (Brasil, 1997), passou a ser registrado no SIH a circunstância do evento no caso de causas externas, destacando tratar-se de um acidente no local de trabalho, acidente de trajeto, outro acidente de trânsito e outras causas externas.

De acordo com o Departamento de Informática do SUS (DATASUS, 2000), dentre as internações hospitalares, as causas externas vêm correspondendo a cerca de 6,0\% em 1998 e 1999. Dentre os óbitos, em 1979 as causas externas representavam $9,2 \%$ passando a $12,7 \%$ em 1998. No Brasil, as causas externas têm sido a sétima causa mais comum de internação hospitalar e a segunda de óbitos, sem se considerar as mortes pelas causas mal definidas, no final da década de 1990.

Ainda segundo os dados do SIH, DATASUS (1998), no Brasil em 1998 os acidentes de trabalho representaram $8,4 \%$ dos eventos internados classificados como causas externas, e na Bahia essa proporção foi de $12,9 \%$, inferiores, portanto, ao encontrado no presente estudo. Ressalta-se, no entanto, que os eventos que levam à internação devem ser os casos mais graves e não necessariamente correspondem ao conjunto dos eventos atendidos nas emergências.

No estudo de Deslandes (1999), citado anteriormente, a proporção de acidentes de trabalho dentre as causas externas foi de $18,7 \%$ num hospital e $15,0 \%$ no outro. Porém, nesse estudo não foram registrados como acidente de trabalho os de trajeto, e a coleta incluiu a noite e os finais de semana, quando as outras causas externas que não os AT devem preponderar. No presente estudo, caso houvesse sido considerado somente os acidentes típicos, a proporção de casos reduziria de $31,6 \%$ para $24,2 \%$, aproximando-se dos valores encontrados por Deslandes.

Em Curitiba, Paraná, num pronto socorro, em um mês de coleta de meio-dia até 20:00 horas, foram identificados 170 acidentes de trabalho. Desses, a proporção de trabalhadores celetistas foi de $58,8 \%$. A autora chamou a atenção que mesmo dentre esses trabalhadores para os quais deveria ser emitida a CAT, o Hospital deixou de solicitar à empresa o registro do 
acidente em mais de $30 \%$ dos casos. Somandose aos demais trabalhadores não cobertos por esse registro, conclui que a CAT não é um bom instrumento para fins de estudos epidemiológicos. Esse estudo não avaliou a participação dos AT dentre as causas externas atendidas (Ventura, 1998).

Ademais, em relação aos acidentes de trabalho fatais, num estudo do período de 1979 a 1988, o número de mortes por acidentes de trabalho registrados nas DOs, equivaleram a $56,8 \%$ dos registros fatais feitos através das CAT da Previdência Social. O esperado seria encontrar mais acidentes fatais, uma vez que nas DO deveriam estar registrados todos os acidentes de trabalho com óbito, inclusive dos trabalhadores do mercado informal (Beraldo et al.,1993). Os dados mais recentes do SIM, demonstram que os totais dos óbitos registrados como acidentes de trabalho nas DO têm permanecido próximos da proporção demonstrada no estudo acima (DATASUS, 1999).

Para fins de comparação, avaliou-se a participação na força de trabalho (população ocupada) na Região Metropolitana de Salvador (RMS), de acordo com os dados de 1998 da Pesquisa Nacional por Amostra de Domicílios (PNAD) (IBGE, 1998), dos principais ramos de atividade dos trabalhadores acidentados. A construção civil representava $8,5 \%$ da força de trabalho ocupada, enquanto que neste estudo $26,5 \%$ dos acidentes ocorreram com trabalhadores desse setor. O comércio representava $16,6 \%$ da força de trabalho e $25,0 \%$ acidentados eram desse setor. A indústria da transformação representava $8,7 \%$ e $14,7 \%$ dos acidentados eram dos seus quadros. A agricultura representava $1,9 \%$ e houve $5,9 \%$ de acidentes com trabalhadores do setor. Quanto aos serviços domésticos ocorreu o inverso, representava $9,9 \%$ da força de trabalho e houve $7,4 \%$ de acidentes com seus trabalhadores. A maior diferença nessa distribuição ocorreu para os trabalhadores da construção civil, o que pode estar indicando um excesso de acidentes.

Quanto à distribuição por setores de atividade econômica dos acidentes típicos e de trajeto, notificados à Previdência Social através das CAT na Bahia em 1999, chamam a atenção alguns resultados distintos. Dentre as notificações, o setor que registrou mais acidentes foi a indústria de transformação (23,3\%), superando a construção civil e o comércio com $13,3 \%$ cada. A agricultura notificou 8,0\% dos acidentes no estado (MPAS, 2000). Esse dado comparado com o encontrado, sugere que a construção civil e o comércio estariam subnotificando mais os acidentes de trabalho, o que justificaria a

\begin{tabular}{|c|c|c|c|}
\hline \multirow[t]{2}{*}{ Variáveis } & \multicolumn{3}{|c|}{ Tipo de vínculo } \\
\hline & $\begin{array}{c}\text { Total de } \\
\text { acidentados } \\
n=68\end{array}$ & $\begin{array}{c}\text { Trabalhadores } \\
\text { formais } 1 \\
n=24\end{array}$ & $\begin{array}{c}\text { Trabalhadores } \\
\text { informais } 2 \\
n=44\end{array}$ \\
\hline \multicolumn{4}{|l|}{ Sexo } \\
\hline Masculino & 89,7 & 95,8 & 86,4 \\
\hline Feminino & 10,3 & 4,2 & 13,6 \\
\hline \multicolumn{4}{|l|}{ Tipo do acidente } \\
\hline Típico & 77,9 & 88,0 & 72,1 \\
\hline Trajeto & 22,1 & 12,0 & 27,9 \\
\hline \multicolumn{4}{|l|}{ Escolaridade } \\
\hline Analfabeto & 5,9 & 4,2 & 6,8 \\
\hline 1o grau incompleto & 69,1 & 66,7 & 70,5 \\
\hline \multicolumn{4}{|l|}{ Principais ramos de atividade } \\
\hline Construção civil & 26,5 & 33,3 & 22,7 \\
\hline Comércio & 25,0 & 29,2 & 20,4 \\
\hline Indústria de transformação & 14,7 & 8,3 & 25,0 \\
\hline
\end{tabular}

1 Trabalhadores celetistas com carteira de trabalho assinada.

2 Autônomos, empregados domésticos e empregados sem carteira assinada.

baixa proporção nas estatísticas da Previdência Social.

Segundo o Ministério da Previdência e Assistência Social (MPAS, 2000), a proporção de acidentes de trajeto dentre o total de acidentes em 1999 foi de 10,3\% para o Brasil e 12,9\% para a Bahia, inferior ao encontrado neste estudo que foi de 22,9\%. Uma possível explicação para esse fato é que a caracterização do acidente no trajeto de ida ou de volta como sendo de trabalho, seja menos comum do que os ocorridos no local de trabalho e/ou a serviço da empresa; portanto os acidentes de trajeto aparecem em menor proporção nas estatísticas oficiais por serem mais subnotificados do que os típicos.

Para fazer uma avaliação se havia sub-registro dos AT encontrados, perguntou-se às empresas se haviam notificado. Das que responderam, somente $45,5 \%$ tinham notificado os AT. Essa estimativa de subnotificação reveste-se de importância se for considerado que grande parte dos acidentes atendidos nessa emergência $(40,2 \%)$, foi de ferimentos graves, politraumatismos e fraturas que devem ter implicado o afastamento do trabalhador e mesmo assim, menos da metade dos acidentes foram notificados.

Importante medida no sentido de melhorar a geração e utilização das informações de saú- 
de dos serviços de emergência e reforçar as ações preventivas das causas externas, ocorreu com a aprovação da Política Nacional de Redução da Morbimortalidade por Acidentes e Violências, já citada. As diretrizes para a consecução dos propósitos da Política, ilustram essa afirmativa, sendo elas: promoção da adoção de comportamentos e de ambientes seguros e saudáveis; monitorização da ocorrência de acidentes e violências; sistematização, ampliação e consolidação do atendimento pré-hospitalar; assistência interdisciplinar e intersetorial às vítimas de acidentes e violências; estruturação e consolidação do atendimento voltado à recuperação e à reabilitação; capacitação de recursos humanos; e apoio ao desenvolvimento de estudos e pesquisas.

Complementa ainda o documento que essa Política prioriza as medidas preventivas, entendidas em seu sentido mais amplo, abrangendo desde as medidas inerentes à promoção da saúde e aquelas voltadas a evitar a ocorrência de violências e acidentes, até aquelas destinadas ao tratamento das vítimas, nestas compreendidas as ações destinadas a impedir as seqüelas e as mortes devidas a estes eventos.
O presente estudo teve um caráter exploratório e o seu desenho não permite extrapolações dos seus resultados. Tem como principal perspectiva subsidiar o SUS na implantação da vigilância das causas externas nas emergências, dentro de um programa de redução da morbimortalidade por causas externas que vem sendo implantado no Estado da Bahia em consonância com a Política Nacional dessa área.

Entende-se que a vigilância dos acidentes de trabalho deve ser realizada em conjunto com a vigilância das demais causas externas, visando o melhor aproveitamento de recursos, observando que incluem-se para esses eventos como estratégias do SUS de prevenção e controle, também as intervenções nos locais de trabalho.

Em concordância com o abordado por Waldman \& Mello-Jorge (1999), conclui-se que as emergências podem ser importante fonte de informação dos acidentes de trabalho, de baixo custo e de simples obtenção. A implantação da vigilância desses eventos nesses locais, será um passo importante para conhecer melhor a realidade de acidentes de trabalho no conjunto dos trabalhadores, ainda que seja necessário o desenvolvimento de pesquisas epidemiológicas para complementação desses resultados.

\section{Referências}

BARATA, R. B.; RIBEIRO, M. C. S. A. \& MORAES, J. C., 2000. Acidentes de trabalho referidos por trabalhadores moradores em área urbana no interior do Estado de São Paulo em 1994. Informe Epidemiológico do SUS, 9:199-210.

BERALDO, P. S. S.; MEDINA, M. G.; BORBA, E. A. \& SILVA, L. P., 1993. Mortalidade por acidentes do trabalho no Brasil - Uma análise das Declarações de Óbito, 1979-1988. Informe Epidemiológico do SUS, 2:41-54.

BRASIL, 1997. Portaria no 142 de 13 de novembro de 1997. Define classificação do caráter da internação em casos compatíveis com causas externas. Brasília: Diário Oficial da República Federativa do Brasil, no 203, p. 10, 17 nov. Seção I.

BRASIL, 2001. Portaria no 737 de 16 de maio de 2001. Aprova a Política Nacional de Redução de morbimortalidade por acidentes e violência. Brasília: Diário Oficial da República Federativa do Brasil, no 96-E, pp. 3-8, 18 maio, Seção I. 
CARVALHO, D. M., 1997. Grandes Sistemas Nacionais de Informação em Saúde: Revisão e discussão da situação atual. Informe Epidemiológico do SUS, 4:7-46.

DATASUS (Departamento de Informática do SUS), 1998. Sistema de Informações Hospitalares. CDROM. Brasília: Ministério da Saúde.

DATASUS (Departamento de Informática do SUS), 1999. Sistema de Informações sobre Mortalidade; 1979-1997: Dados de Declaração de Óbito. CDROM. Brasília: Ministério da Saúde.

DATASUS (Departamento de Informática do SUS), 2000. Informações em Saúde. 10 Março $2002<$ http: //www.datasus.gov.br>.

DESLANDES, S. F., 1999. O atendimento às vítimas de violência na emergência: "Prevenção numa hora dessas?" Ciência \& Saúde Coletiva, 4:81-94.

DRUMOND Jr., M.; LIRA, M. M. T. A.; FREITAS, M.; NITRINI, T. M. V. \& SHIBAO, K., 1999. Avaliação da qualidade das informações de mortalidade por acidentes não especificados e eventos com intenção indeterminada. Revista de Saúde Pública, 33: 273-280.

IBGE (Fundação Instituto Brasileiro de Geografia e Estatística), 1998. Pesquisa Nacional por Amostra de Domicílios, v. 20, Bahia e Região Metropolitana de Salvador. Rio de Janeiro: IBGE.
IBGE (Fundação Instituto Brasileiro de Geografia e Estatística), 2000. Pesquisa Nacional por Amostra de Domicílios. 10 Março 2002 <http://www.ibge. gov.br>.

MENDES, A. C. G.; SILVA Jr., J. B.; MEDEIROS, K. R.; LYRA, T. M.; MELO FILHO, D. \& SÁ, D. A., 2000. Avaliação do Sistema de Informações Hospitalares - SIH/SUS como fonte complementar na vigilância e monitoramento de doenças de notificação compulsória. Informe Epidemiológico do SUS, 9:67-86.

MPAS (Ministério da Previdência e Assistência Social), 2000. Anuário Estatístico da Previdência Social 1999. 10 Março 2002 <http://www.mpas.gov.br>.

OMS (Organização Mundial da Saúde), 1995. Classificação Estatística Internacional de Doenças e Problemas Relacionados à Saúde, 10a Revisão. v. 1. São Paulo: Centro Colaborador da OMS para a Classificação de Doenças em Português.

VENTURA, M. T. S. M., 1998. Notificação de acidente de trabalho em pronto-socorro de Curitiba. Boletim Informativo do CEMAST/PR, 3:2.

WALDMAN, E. A. \& MELLO-JORGE, M. H. 1999. Vigilância para acidentes e violência: Instrumento para estratégias de prevenção e controle. Ciência \& Saúde Coletiva, 4:71-79.

Recebido em 25 de março de 2001

Versão final reapresentada em 28 de maio de 2002

Aprovado em 8 de julho de 2002 